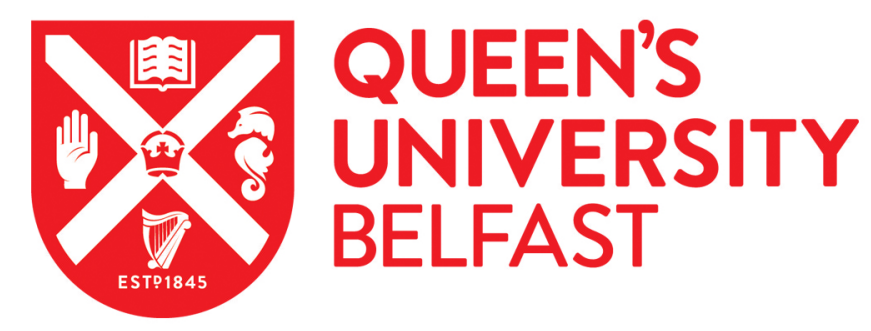

\title{
Changes in zinc status and zinc transporters expression in whole blood of patients with Systemic Inflammatory Response Syndrome (SIRS)
}

Florea, D., Molina-López, J., Hogstrand, C., Lengyel, I., de la Cruz, A. P., Rodríguez-Elvira, M., \& Planells, E. (2017). Changes in zinc status and zinc transporters expression in whole blood of patients with Systemic Inflammatory Response Syndrome (SIRS). Journal of trace elements in medicine and biology : organ of the Society for Minerals and Trace Elements (GMS). https://doi.org/10.1016/j.jtemb.2017.11.013

Published in:

Journal of trace elements in medicine and biology : organ of the Society for Minerals and Trace Elements (GMS)

Document Version:

Peer reviewed version

Queen's University Belfast - Research Portal:

Link to publication record in Queen's University Belfast Research Portal

\section{Publisher rights}

Copyright 2018 Elsevier Ltd.

This manuscript is distributed under a Creative Commons Attribution-NonCommercial-NoDerivs License

(https://creativecommons.org/licenses/by-nc-nd/4.0/), which permits distribution and reproduction for non-commercial purposes, provided the author and source are cited.

\section{General rights}

Copyright for the publications made accessible via the Queen's University Belfast Research Portal is retained by the author(s) and / or other copyright owners and it is a condition of accessing these publications that users recognise and abide by the legal requirements associated with these rights.

Take down policy

The Research Portal is Queen's institutional repository that provides access to Queen's research output. Every effort has been made to ensure that content in the Research Portal does not infringe any person's rights, or applicable UK laws. If you discover content in the

Research Portal that you believe breaches copyright or violates any law, please contact openaccess@qub.ac.uk. 


\title{
Manuscript Details
}

\section{Manuscript number}

Title
JTEMB_2017_444

Changes in expression of zinc transporters in whole blood of patients with Systemic Inflammatory Response Syndrome (SIRS)

Article type

Research Paper

\begin{abstract}
Introduction: Critically ill patients develop severe stress, inflammation and a clinical state that may raise the utilization and metabolic replacement of many nutrients and especially zinc, depleting their body reserves. This study was designed to assess the zinc status in critical care patients with systemic inflammatory response syndrome (SIRS), comparing them with a group of healthy people, and studying the association with expression of zinc transporters. Material and methods: This investigation was a prospective, multicentre, comparative, observational and analytic study. Twelve critically ill patients from different hospitals and 12 healthy subjects from Granada, Spain, all with informed consent were recruited. Data on daily nutritional assessment, ICU severity scores, inflammation, clinical and nutritional parameters, plasma and blood cell zinc concentrations, and levels of transcripts for zinc transporters in whole blood were taken at admission and at the seventh day of the ICU stay. Results: Zinc levels on critical ill patient are diminish comparing with the healthy control (HS: $0.94 \square 0.19$; CIPF: $0.67 \square 0.16 \mathrm{mg} / \mathrm{dL}$ ). The 58 percent of critical ill patients showed Zinc plasma deficiency at beginning of study while 50.0 percent of critical ill after 7 days of ICU stay. ZnT7, ZIP4 and ZIP9 were the zinc transporters with highest expression in whole blood. In general, all Zinc transporters were significantly down-regulated $(P<0.05)$ in the critical ill population at admission in comparison with healthy subjects. Severity scores and inflammation were significantly associated $(P<0.05)$ with zinc plasma levels, and zin transporters ZIP3, ZIP4, ZIP8, ZnT6, Znt7. Expression of 11 out of 24 zinc transporters was analysed, and $\mathrm{ZnT1}, \mathrm{ZnT4}, \mathrm{ZnT5}$ and ZIP4, which were downregulated by more than 3-fold in whole blood of patients. Conclusion: In summary, in our study an alteration of zinc status was related with the severity-of-illness scores and inflammation in critical ill patients since admission in ICU stay. SIRS caused a general shut-down of expression of zinc transporters in whole blood. That behavior was associated with severity and inflammation of patients at ICU admission regardless zinc status. We conclude that zinc transporters in blood might be useful indicators of severity of systemic inflammation and outcome for critically ill patients.
\end{abstract}

Keywords

Manuscript category

Corresponding Author

Corresponding Author's Institution

Order of Authors

Suggested reviewers
Zinc level; Critically ill patients; Systemic Inflammatory Response Syndrome (SIRS); Zinc transporters; Severity.

Clinical studies

Elena Planells

Department of Physiology, Institute of Nutrition and Food Technology "José Mataix". Biomedical Research Center. Health Campus. University of Granada. 18071. Granada

Daniela Florea, Jorge Molina-López, Christer Hogstrand, Imre Lengyel, Antonio Pérez de la Cruz, Manuel Rodríguez-Elvira, Elena Planells

Antonio Ayala, Vojtech Adam 


\section{Submission Files Included in this PDF}

File Name [File Type]

Paper_30_10_2017 to JTEBM.doc [Manuscript File]

Fig.1.tiff [Figure]

Fig.2.tiff [Figure]

Fig.3.tiff [Figure]

Fig.4.tiff [Figure]

Fig.5.tiff [Figure]

Table 1.docx [Table]

Table 2.docx [Table]

Table 3.docx [Table]

Conflicts of Interest.docx [Conflict of Interest]

To view all the submission files, including those not included in the PDF, click on the manuscript title on your EVISE Homepage, then click 'Download zip file'. 
1 Changes in expression of zinc transporters in whole blood of patients with

2 Systemic Inflammatory Response Syndrome (SIRS)

3

$4 \quad \mathrm{PhD}$. Daniela Florea 1,2

$5 \quad$ PhD. Jorge Molina-López ${ }^{1}$

6 Prof PhD Christer Hogstrand ${ }^{3}$

$7 \quad$ PhD Imre Lengyel 4

8 Dr. $\mathrm{PhD}$. Antonio Pérez de la Cruz ${ }^{5}$

9 Dr. Manuel Rodríguez-Elvira ${ }^{6}$

10 Prof. PhD. Elena Planells ${ }^{1 *}$ manuel.rodriguez.elvira.sspa@juntadeandalucia.es. 
* Corresponding author

Christer Hogstrand, Metal Metabolism Group, Diabetes and Nutritional Sciences, King's College London, 3.85 Franklin-Wilkins Building, 150 Stamford Street, London SE1 9NH; Email: christer.hogstrand@kcl.ac.uk, Tel: 44 (0)20 7848 4436, Fax: 44 (0)20 78484171

Elena María Planells del Pozo, Department of Physiology, University of Granada, Faculty of Pharmacy, Biomedical Research Center, Institute of Nutrition and Food Technology "José Mataix", Avda del Conocimiento S/N, 18071 Granada, Spain, European Union; E-mail: elenamp@ugr.es; phone: +34-958241000 EXT 20313

\section{List of Abbreviations}

Intensive Care Unit (ICU); Systemic Inflammatory Response Syndrome (SIRS); Acute Physiology and Chronic Health Evaluation (APACHE II); Sequential Organ Failure Assessment (SOFA); Solute Carrier Family 30 (ZNT/SCL30A (1-10)); Solute Carrier Family 39 (ZIP/SCL39A (1-14)); Healthy Subject (HS); Critically ill patients (CIP); Critically ill patient baseline (CIPB); Critically ill patient 7days (CIP7); Enteral nutrition (EN); Parenteral nutrition $(\mathrm{PN}), \mathrm{CHO}$ (carbohydrates); C-reactive protein (CRP)

\section{Acknowledgments}

We thank all (Virgen de las Nieves, San Cecilio, General of Baza and Santa Ana of Motril, Granada) hospital workers, especially ICU and Clinical Analysis Service personnel. We also thank the Supported Unit for Investigation FIBAO (Foundation for the Health Investigation). Financial support for the study was provided by Project FIS PI10/1993 from the Spanish Carlos III Health Institute and FEDER European Funds. 
Introduction: Critically ill patients develop severe stress, inflammation and a clinical state that may raise the utilization and metabolic replacement of many nutrients and especially zinc, depleting their body reserves. This study was designed to assess the zinc status in critical care patients with systemic inflammatory response syndrome (SIRS), comparing them with a group of healthy people, and studying the association with expression of zinc transporters.

Material and methods: This investigation was a prospective, multicentre, comparative, observational and analytic study. Twelve critically ill patients from different hospitals and 12 healthy subjects from Granada, Spain, all with informed consent were recruited. Data on daily nutritional assessment, ICU severity scores, inflammation, clinical and nutritional parameters, plasma and blood cell zinc concentrations, and levels of transcripts for zinc transporters in whole blood were taken at admission and at the seventh day of the ICU stay.

Results: Zinc levels on critical ill patient are diminish comparing with the healthy control (HS: $0.94 \pm 0.19$; CIPF: $0.67 \pm 0.16 \mathrm{mg} / \mathrm{dL}$ ). The 58 percent of critical ill patients showed Zinc plasma deficiency at beginning of study while 50.0 percent of critical ill after 7 days of ICU stay. ZnT7, ZIP4 and ZIP9 were the zinc transporters with highest expression in whole blood. In general, all Zinc transporters were significantly down-regulated $(\mathrm{P}<0.05)$ in the critical ill population at admission in comparison with healthy subjects. Severity scores and inflammation were significantly associated $(\mathrm{P}<$ 0.05) with zinc plasma levels, and zin transporters ZIP3, ZIP4, ZIP8, ZnT6, Znt7. Expression of 11 out of 24 zinc transporters was analysed, and ZnT1, ZnT4, ZnT5 and ZIP4, which were downregulated by more than 3-fold in whole blood of patients.

Conclusion: In summary, in our study an alteration of zinc status was related with the severity-of-illness scores and inflammation in critical ill patients since admission in ICU stay. SIRS caused a general shut-down of expression of zinc transporters in whole 
blood. That behavior was associated with severity and inflammation of patients at ICU admission regardless zinc status. We conclude that zinc transporters in blood might be useful indicators of severity of systemic inflammation and outcome for critically ill patients.

\section{Keywords}

Zinc level; Critically ill patients; Systemic Inflammatory Response Syndrome (SIRS); Zinc transporters; Severity.

\section{Highlights}

Critical ill patients are deficient in plasma and cellular zinc.

Down-regulation of zinc transporters linked to critical illness.

New zinc transporters as biomarker candidates for severity and inflammation in critical ill patients.

Introduction

Zinc is an essential component in all levels of metabolism, and is a key component in structure of genetic material [1]. Zinc homeostasis depends on the balance between absorption of zinc from diet and endogenous secretions by the daily intake of the element [2]. These basic conditions may be complicated by diseases or dysfunctions as multiple organ failure that in critically ill patients require a correct and timely provision of energy and nutrients as zinc that can save life, so adequate nutrition should be included in their clinical treatment [3, 4]. In critically ill patients, low zinc concentrations have been reported linked to oxidative stress, systemic inflammatory response syndrome (SIRS) and immune disorders [4, 5], particularly in patients with sepsis [6-9]. Then, zinc deficiency could be considered as an important factor in the pathogenesis of different diseases [5]. Nevertheless, increased zinc demands are 
justified by hypercatabolic situation and a high degree of stress as surgical, traumatic and septic shock, which could develop malnutrition status [10].

Zinc is an element very difficult to be determined by non-invasive techniques in human body tissues, therefore its represents a challenge in the determination of a suitable biomarker of zinc status for critical condition. Although studies concluded that plasma, urinary and hair zinc are reliable biomarkers of zinc status [11], there is no consensus. Recently, Lowe et. al. [12], concluded that zinc is mostly determined in plasma or serum samples. Nevertheless, peripheral blood tissue is an accessible tissue to use for the exploration of biomarkers for human zinc homeostasis [13] but cellular blood zinc needs further investigation [12]. Over the time has been studied to find other biomarkers for zinc status in human body [11] on these days many of research is concentrated on zinc transporters. Some authors suggest that zinc transporters could express independently of dietary or plasma zinc in healthy individuals [14]. On the other hand, the dysregulation of expression and activity of ZIP and ZnT transporters involved in the pathogenesis and progression in chronic diseases have been described [15]. However, their molecular relationship with the etiology of acute diseases is far from complete. Clarifying these issues would lead to therapeutic innovation in critical illness, and therefore, great attention should be paid to ZIP and ZnT transporters.

There are no previous studies that were comprehensively addressed the zinc transporters levels in the cell membrane in humans. Our aim was to investigate the zinc concentrations and its association with the expression level of all zinc transporters in blood from healthy and critically ill individuals and determine their differences in expression. We also want to explore the relationship among zinc expression transporter levels in blood and the severity situation in critical ill condition. It is indispensable to find a biomarker for zinc status in order to ameliorate the evolution of critical ill patient and to optimize their clinical intervention during their ICU stay. 


\section{Material and methods}

\section{$\underline{\text { Study design }}$}

The study design is based on a prospective, multicentre, observational and analytic study, monitoring the critically ill patient, from admission until the seventh day of ICU stay, from different hospitals of Southern Spain (Virgen de las Nieves, San Cecilio, General of Baza and Santa Ana of Motril, Granada). The study was carried out according to the code of ethics of the World Medical Association (Declaration of Helsinki) for experiments involving humans and the International Conference on Harmonization/Good Clinical Practice Standards. Informed consent was obtained from patients or family who had agreed to participate in the study, considering the approval of the Ethics Committee and the Research Committee of the Centre involved.

\section{$\underline{\text { Participants }}$}

A total of 12 and 12 subjects were included in healthy control (HS) and critically ill groups, respectively (CIPB, critical ill patients on admission; CIPF critical ill patient follow-up), aged and gender matched. Inclusion criteria for the healthy population were: not to present any alteration that could have influence on their nutritional status and to have agreed to form part of the study. In critical ill population, the study was carried out with consecutive patients admitted to ICU. The criteria to be included in the study were: critically ill patients older than 18 years, admitted in the ICU; with SIRS and APACHE II score $>15$; to have artificial nutritional support (enteral and mixed enteral and parenteral nutrition); to present no neurological, muscular, skeletal, or situations that affected the mouth or upper digestive tract or contraindicate the passage of nutrients to the other portions of the digestive system; and to continue in the Unit for at least 7 days. Exclusion criteria were: refusal by the patient or their legal representatives to participate in the study; pregnancy; highly contagious disease; allergies; cancer; HIV; food orally intake before obtaining the analytical sample at baseline; intolerance or contraindication for using enteral route. Clinical characteristics as age, sex and diagnosis were recorded 
at study enrolment (ICU admission). SIRS, the Acute Physiology and Chronic Health Evaluation (APACHE II) and the Sequential Organ Failure Assessment (SOFA) scores and mortality was included at admission and seven days in ICU stay.

\section{Nutritional profile}

Nutritional intake profile in healthy population was made through personal interview by trained staff in the use of nutritional techniques, employing questionnaires with the following sections: personal data and consumption habits, the nutritional intake by $72 \mathrm{~h}$ recall and food frequency consumption (FFQ) questionnaires. Nutrient intake and adequacy were calculated with the Nutriber ${ }^{\circledR}$ software, comparing with the Recommended Dietary Allowances (RDAs) for healthy population [16]. Nutritional support protocol in critical ill patients was assessed according to the Clinical Nutrition Units of hospitals, based on American Society for Parenteral and Enteral Nutrition and the European Society of Parenteral and Enteral Nutrition Guidelines [17]. All patients received nutritional standard support via enteral, parenteral or combined, administrating nutritional formulas elaborated in the pharmacies of the hospitals or from commercial products. A daily nutritional log was kept for each patient (type, volume and composition of intake, tolerance, among other factors) from admission to seven days in ICU. Zinc support was daily calculated and registered by the nutritionists and represented as the average of a seven-day period of stay the ICU.

\section{$\underline{\text { Biochemical parameters }}$}

Fasting blood samples $(10 \mathrm{~mL})$ were drawn from ICU patients (between the hours of 08:30 am and 10:00 am during fasting) by venepuncture after the hemodynamic stabilization phase of admission and after 7 days of stay. The same methodology was applied for healthy subjects for biochemical testing. Vacutainer tubes (Venoject ${ }^{\circledR}, \mathrm{BD}$, UK) containing a solution of heparin of lithium as anticoagulant were used. Samples were centrifuged at $3000 \mathrm{RPM}$ for 15 minutes at $4^{\circ} \mathrm{C}$. Blood drawn was centrifuged to separate plasma and cells and stored at $-80{ }^{\circ} \mathrm{C}$. Biochemical variables such as glucose, 
albumin, prealbumin, urea, uric acid, alkaline phosphatase, CPK, C-reactive protein (CRP), rheumatoid factor, total protein, transferrin, homocysteine, leucocytes, cooper and iron concentrations were determined by the hospital laboratory using standard techniques and following the quality control and established procedures.

\section{$\underline{\text { Plasma and cellular zinc }}$}

Plasma and cellular zinc was determined in wet-mineralized triplicate samples $[18,19]$. Inductively couple plasma mass spectrometer (ICP, Agilent, UK) was selected for plasma zinc analysis. All the variables were taken at day 0 and day 7 calculating for each variable the mean difference assuming a maximum error of 5\% and a confidence level of $95 \%$.

\section{$\underline{\text { Gene expression }}$}

\section{$\underline{\text { RNA isolation }}$}

Blood and RNA Preparation $2.5 \mathrm{ml}$ of human peripheral blood was stored in PAXgene ${ }^{\mathrm{TM}}$ whole-blood RNA tubes (Preanalytix) and separated following standard methodology (Qiagen) before storage at $-80^{\circ} \mathrm{C}$. Total RNA was extracted and purified using PAXgene ${ }^{\mathrm{TM}}$ Blood RNA Kit (Qiagen). $1 \mu \mathrm{g}$ of total RNA was reverse transcribed to generate complementary DNA by ThermoScript RNase H-Reverse Transcriptase (Invitrogen Life Technologies, Carlsbad, CA) followed by dilution (1:10) with RNasefree water. The complementary DNA was subjected to quantitative polymerase chain reaction (PCR) analysis by using SYBR Green PCR Master Mix and a HT7900 sequence detection system (Applied Biosystems). $\Delta \mathrm{Ct}$ values were used for all statistical analyses and then transformed to a relative copy number $(\mathrm{RCN})$ value to facilitate the ease of data interpretation. The $\mathrm{RCN}$ of selected genes were determined by normalization to the expression of the 3 housekeeping genes: Glyceraldehyde 3Phosphate Dehydrogenase (GAPDH), Tyrosine 3-monooxygenase/tryptophan 5monooxygenase activation protein (YWHAZ) and Ubiquitin $\mathrm{C}(\mathrm{UBC}) . \mathrm{RCN}=\mathrm{E}-\Delta \mathrm{Ct} \mathrm{x}$ 
$216 \mathrm{Ct}$ reference (the average of 3 housekeeping genes). PCR primer pairs, including those

217 for all known human zinc transporters, were previously validated and reported [7]. Zinc transporters with $\Delta \mathrm{CT}>13$ were considered there are not expressed, or the expression is too low to be detected and were not included in the statistical analysis.

\section{$220 \quad$ Statistical analysis}

Statistical analysis was performed with version 17.0 of the Statistical Package for Social Sciences (SPSS, Chicago, IL, USA). Descriptive statistics were computed for the characteristics of the study population and the biomechanical parameters. Data were expressed as means and standard deviation (SD). For continuous variables, the assumption of normality was tested using the Shapiro Wilk curve-fitting test. The differences between HS and CIP groups were evaluated by using the normal standardized range using Student's t test. The paired t-test was used to compare the evolution of the critical patient in ICU. Bivariate correlation was used to evaluate plasma and cellular zinc concentrations and severity scores with the expression of zinc transporters using the Pearson correlation coefficient. The severity and inflammation variables were dichotomized by the median in order to study their influence on the plasma and cellular zinc status, the zinc-albumin ratio, and the expression of the zinc transporters. The differences between the groups for these parameters were compared using the paired t-test. Data are presented as box-and-whisker plots showing median, interquartile, and full range or individual data points. The level of significance was set at 0.05 .

\section{Results}

Table 1 summarizes the study population characteristics, the severity scores and the diagnosis of the critical population in the ICU. The critical care population showed no significant differences in comparison to the healthy subject control population in 
energy, macronutrient and socio-demographic variables studied. The most frequent diseases presented by the patients on ICU admission were respiratory (33.4 percent), cardiovascular (33.3 percent) and abdominal (33.3 percent). APACHE and SOFA scores showed an improvement thorough the ICU stay being significant for the APACHE score at 7 days of ICU stay $(\mathrm{P}=0.03)$. About energy and macronutrients support, our results showed an energy support was below $2 / 3$ of recommendations in 14 percent of healthy controls against the $60 \%$ of critical ill. Critical ill patient received significantly less $\mathrm{CHO}$ support than control through the study period $(\mathrm{P}<0.01)$. In critical ill patients, all patients had artificial nutrition: EN was received by $50 \%$ of the critical care patients, PN by 33 percent and mixed nutrition $(\mathrm{EN}+\mathrm{PN})$ by 17 percent of patients.

Table 2 summarizes the biochemical values of the study population. During the period studied, clinical parameters were into the reference values for the healthy population. In contrast, critically ill condition showed significant differences $(\mathrm{P}<0.05)$ in clinical and nutritional parameters in comparison with the healthy subjects. All parameters in critically ill patients remained without changes during ICU stay excluding uric acid, creatinine and CRP $(\mathrm{p}<0.05)$.

Fig. 1 shows the zinc status in accordance to zinc supply (Fig. 1A), the plasma zinc levels (Fig. 1B) and cellular zinc (Fig. 1C) in healthy controls and critically ill patients. Regarding, zinc supply no significant differences were found between healthy controls and the critical ill on EN or EN + PN (Fig. 1A). Moreover, there were no patients with zinc supply below the recommendations on EN when the limit was set in $8 \mathrm{mg}$ per day. Nevertheless, a total of 50 percent of patients with PN presented an insufficient zinc supply. In relation to plasma (Fig. 1B) and cellular zinc (Fig. 1C), significant changes were found between critical ill patients at seven days in ICU stay and healthy controls $(\mathrm{p}<0.05)$. We analysed the contribution of each compartment to the total amount of zinc in blood. The healthy controls presented a total of 86.0 percent of the zinc content 
in blood cells and 13.2 percent in plasma. The critical ill population presented a 25.2 percent of the zinc in plasma at admission in ICU and 27.3 percent in plasma after 7 days in ICU stay. Moreover, the 58 percent of critical ill patients showed zinc plasma deficiency at beginning of study while 50.0 percent of critical ill after 7 days of ICU stay.

Fig. 2A represents the heat map showing an overview of the $\mathrm{Ct}$ quality categories that have been assigned to the different groups. In healthy subjects, ZnT7 genes, ZIP1 and ZIP 9 and ZIP4 showed the highest expression profile. In contrast, low or no expression was detected for ZnT2, ZnT3, ZnT8, ZnT10, ZIP2 and ZIP12 genes. In the critical ill patients group (both at admission and at 7 days in ICU stay) low or no expression was detected of, ZnT2, ZnT3, ZnT4, ZnT8, ZnT10, ZIP2, ZIP5, ZIP6, and ZIP12 genes. From the futures analysis we had excluded ZnT2, ZnT3, ZnT4, ZnT8, ZnT10, ZIP2 and ZIP12. For ZnT9, ZIP5, ZIP 6, ZIP11 and ZIP14 the expression is borderline, or the expression is just in one group and diapering form another group. The relative expression profile was calculated to compare the healthy subjects with respect to critical ill patients at admission in ICU (Fig. 2B) and critical ill patients at two time points measured (Fig. 2C). In general, all zinc transporters were significantly down-regulated $(\mathrm{P}<0.05)$ in the critical ill population at admission in comparison with healthy subjects. In contrast, when comparing critical ill patients at admission with 7 days in ICU stay, ZIP1 was the only one statistically significant $(\mathrm{P}<0.01)$. Expression of 11 out of 24 zinc transporters was analysed, and ZnT1, ZnT4, ZnT5 and ZIP4, which were downregulated by more than 3 -fold in whole blood of patients compared to healthy controls.

Based on the observation that the expression of zinc transporters decreased in conjunction with a critical condition we evaluated the zinc transporters expression in the context of severity-of-illness scores and zinc status (Table 3). An increased ZIP1 and ZnT7 expression was directly correlated $(\mathrm{p}<0.05)$ with the APACHE scores on 
admission in ICU. Moreover, ZIP7 and ZIP14, and ZIP10 zinc transporters were significantly associated $(\mathrm{P}<0.05)$ with SOFA score on admission and thorough ICU stay, respectively. Finally, an additional evaluation was conducted to analyse the association regarding zinc status and zinc plasma levels. An indirect relationship observed $(\mathrm{P}<0.05)$ between the status of zinc in plasma and the ZIP13 and ZIP14 expression during the follow-up study. Finally, the relationship between the status of zinc in blood was explored. Plasma zinc concentrations were directly correlated $(\mathrm{P}<$ $0.05)$ to cellular zinc content in critically ill on admission in ICU.

Because circulating zinc is primarily bound to albumin, and because albumin levels were significantly decreased in critically ill patients on admission $(\mathrm{P}=0.01)$ and seven days in ICU stay $(\mathrm{P}=0.49)$ compared to healthy controls, we calculated the ratio of plasma zinc to albumin. The mean zinc/albumin ratio for critical ill patients was $0.28 \pm$ $0.12 \mathrm{mg} / \mathrm{dL}$ on admission and $0.29 \pm 0.06 \mathrm{mg} / \mathrm{dL}$ at seventh day in ICU stay (Fig. 3). However, no statistical significant differences were found for critical ill comparison during the follow up study and inflammation and severity scores.

Concerning the relationship between severity and inflammation with the altered clinical parameters in the critical patient, we decided to study whether presenting a higher level of inflammation or severity significantly affected the expression of zinc transporters (Fig. 4). In fact, severity-of-illness scores and inflammation were dichotomized by the median. Related to the status of zinc, critical ill patients with high severity $(\mathrm{P}=0.021)$ and inflammation $(\mathrm{P}=0.048)$ presented significantly less zinc in plasma compared to the less severe patients (Fig. 4A). At the same time, a higher severity and inflammation resulted in a lower expression patterns of the ZIP3 $(\mathrm{P}=0.016), \mathrm{ZnT} 6(\mathrm{P}=0.003), \mathrm{ZIP} 8$ $(\mathrm{P}=0.037), \mathrm{ZnT} 7(\mathrm{P}=0.043)$ and ZIP4 $(\mathrm{P}=0.048)$ transporters at admission ICU (Fig. 4B, Fig. 4C and Fig. 4D). Finally, Fig. 5 shows the RNA expression of ZIP8 in healthy controls and critically ill patients on admission and within the follow-up study. Inter- 
groups comparison showed significantly less ZIP8 expresion in critical ill population (P $=0.012)$ and healthy controls.

\section{Discussion}

The main finding of the present study showed a relationship between inflammation and severity with zinc in plasma in critical ill patients through ICU stay. A novelty in our study was the assessment of the expression profile of all zinc transporters. It is important to mention that our results showed a diminished expression profile in all zinc transporters mainly due to the critical ill condition. In fact, the decrease in expression of certain zinc transporters was found to be associated with severity and inflammation of patients at ICU admission regardless of plasma and cellular zinc status. Based on these considerations and the difficulty of assessing nutritional status in severely ill subjects, the design of our study allowed us to determine the relationship between zinc metabolic status with inflammation and severity suggesting that the critical condition was correlated with a decrease in the functionality of zinc transporters.

About critical illness, a high risk of morbidity and mortality was observed considering the hyper-catabolic situation due to the high APACHE II and SOFA scores. With regards to energy and macronutrients support, the control of nutrition support in critically ill patient is necessary for the maintenance and recover of organic function expenses [17]. The methodology to predict resting energy expenditure are questionable in critically ill patients is still difficult to adjust requirements during ICU stay [20]. Considering the nutritional supply, our results showed no patients with zinc insufficient supply by EN and PN (2 patients). In general, we did not find significant differences in zinc supply between groups (HS vs. CIP) and we could consider zinc supply in critical ill was adequate. Confirming that, several studies demonstrated that mixed EN and PN supply does not derive nutrient deficiency in critical ill patients [21, 22]. 
Low plasma zinc has been reported previously in ICU populations [4, 6, 9]. In our study the majority of blood parameters were more affected in ICU patients with sepsis, both admission and through the ICU stay compared to healthy controls as previous suggested [22]. We found that critical ill patients presented low zinc plasma levels during ICU stay. Whereas we did not find significant differences between the control population and critical patients at admission, we found that 58 percent of the patients presented deficiencies of plasma zinc. Also, a significant negative association in zinc plasma concentrations with the severity of illness was observed in the sepsis cohort based on SOFA score (Fig. 4A), showing that the most severe patients were those with lower plasma zinc. These findings suggested that perturbations in zinc metabolism were enhanced in the setting of severe infection. In addition, zinc concentrations predictably declined with increased severity of illness scores, which was consistent with a study that was recently conducted in a critically ill paediatric cohort [23]. Other studies [23, 24] have also reported that the extent of the decline in plasma zinc concentrations was associated with increased organ failure and mortality suggesting that the zinc redistribution may be a compensatory mechanism or an indication of declining function and worsening outcomes.

The exact mechanisms by which the decrease in plasma zinc in critical illness occurs are not clear. It has been postulated that compartmental redistribution of zinc from the circulation to tissues as part of the acute phase response, resulting in low plasma concentrations of zinc, is thought to be mediated in part by inflammation, [23] where zinc is required for synthesis of acute phase proteins [25]. According to that, in our study, when dichotomized groups by C-reactive protein, patients with higher C-reactive protein showed significantly lower plasma zinc levels on admission (Fig. 4A). We also found a tendency to statistical significance $(\mathrm{P}=0.078)$ between levels of $\mathrm{C}$-reactive protein and plasma zinc status of critical patients at 7 days of ICU stay once patients were stabilized and reached greater homogeneity as a group. In this sense, as other 
authors recently reported, low zinc concentrations has also been associated with inflammation of the critical ill patient [9].

Albumin is a zinc binding protein which levels were significantly decreased in our critically ill patients on ICU admission and at seven days of ICU stay compared to healthy controls. Low plasma zinc could be due, at least in part, to a drop-in albumin levels. Circulating zinc is primarily albumin bound, and thus zinc concentrations are dependent to some degree on serum albumin levels [23]. In our study, serum zinc levels and zinc:albumin ratio of the patients were found to be below the reference ranges on admission to the ICU. In accordance to Cander et al. [6], similar zinc:albumin ratio was found for critical illness reference (Fig. 3). On the other hand, we found a direct relationship between the cellular zinc:albumin ratio and the inflammation $(\mathrm{r}=0.757$; $\mathrm{P}$ $=0.011$ ) for critical ill patients during the follow-up study. Several authors have suggested that in plasma, zinc is primarily bound to albumin, but the primary functions of zinc are carried out at the intracellular level [23]. In this sense, the inflammation could be responsible for the mobilization of zinc at intracellular level due to the higher cellular zinc:albumin ratio in critical ill at seventh day in ICU stay.

Zinc is vital to many cellular functions, including protein synthesis, signal transduction, and gene transcription and is necessary to maintain proper immune function. Zinc transporters comprises the SLC30 and SLC39 family members exhibit tissue-specific expression and respond differently in human health and diseases [26]. Moreover, dysregulation of expression and activity of both transporters has been suggested to be involved in the pathogenesis and progression of several diseases although comprehensive understanding is far from complete [26]. It is indispensable to find a biomarker in blood for zinc status to ameliorate the evolution of critical ill patient and to optimize their clinical intervention through the ICU stay. Once of our main purposes was to explore the relationship among zinc expression of transporter levels in blood and the severity of critical ill condition. Have been described previously that the regulation 
of zinc transporters like ZIP8 [4] and were mainly influenced by severity and inflammation, respectively. However, we hypothesized that not only those would be affected by the critical condition. Based on this, we observed that expression of the two families of zinc transporters, if expressed at all, was altered in total blood during the early stages of critical illness or sepsis (at admission) compared to the expression profile in healthy subjects (Fig. 2). The inter groups comparison (CIPB vs HS) revealed that most of the zinc transporters were found to be significantly down-regulated in critical ill patients at the admission in ICU. This could suggest that the critical condition could favor the decrease on the expression patterns of zinc transporters because of the inflammation and severity in critical ill patients (Fig. 4).

There are many studies about zinc transporters but none to determine all zinc transporters and level of expression of each one in the blood sample. In our study, the most expressed zinc transporter in both groups was ZnT7, despite it showed a decline of expression in critical ill patients. We found a positive correlation between $\mathrm{ZnT7}$ expression and APACHE score at admission in ICU (Table 3). On the other hand, regarding ZnT6, ZnT7 and ZIP3 transporters, when APACHE variable was dichotomized, we found significantly lower transporter expression in patients with higher APACHE (Fig. 4B and Fig. 4C). From ZnT family transporters as ZnT1, ZnT5, ZnT6 and ZnT9 had previously described in blood $[1,14]$. In the case of ZnT7, a protective role has been shown in reducing oxidative stress, inflammation and cellular apoptosis $[3,27]$.This seems to be in agreement with our results since we studied patients with inflammation possibly caused by oxidative stress. ZnT7 is found in secretory apparatus are responsible for loading zinc to alkaline phosphatases [28] which has been found that the enzyme to reduce inflammation through dephosphorylation and thereby detoxification of endotoxin (lipopolysaccharide), which is an important mediator of sepsis [29]. 
Regarding ZIP family, we observed in ZIP3, ZIP4 and ZIP8 transporters that, when Creactive protein variable was dichotomized, significantly diminished transporter expression in patients with higher C-reactive protein (Fig. 4B and Fig. 4D), probably due to their relationship with inflammation [30]. In our study, ZIP1 resulted a downregulated significantly $(\mathrm{P}=0.007)$, decreasing its expression after 7 days of ICU stay. We also found a positive significant correlation between ZIP1 and APACHE score $\mathrm{P}<$ 0.05 at the baseline. In critically ill patients the expression of ZIP8 and ZIP9 was significantly decreased in comparison with healthy subjects (Fig. 2A). Contrary to Besecker et al. [4], in our case we found a decrease in the expression of ZIP8 mainly associated with a greater inflammation at the admission of the ICU stay. This trend is consistent with the response of other transporters equally analysed in our study. ZIP8 might be a potential biomarker for zinc status [4] but in this study, we suggested adding other zinc transporters including ZIP1, ZIP3, ZIP4, ZIP9, ZnT6 and ZnT7 due to their high expression in blood and their relation to critical condition.

Finding a biomarker for zinc status is mandatory. There is a current appreciated effort from the different zinc research groups working together on finding the biological marker for zinc status. In critical ill disease where the metabolism is disrupted, biochemical parameters are altered cellular and plasma zinc can lead to zinc deficiency. Then, optimizing nutrition for these patients should be included on clinical protocols. The SIRS and APACHE and SOFA high scores presented in our patients, supposed an acute inflammatory status that developed an alteration in the zinc metabolism associated with severity.

To our knowledge, this study is the first to investigate the zinc concentrations in conjunction with the expression level of all zinc transporters in blood from healthy and critically ill individuals and determine their differences in expression. Although our study is based on a relatively small sample, the results have been able to show a significant response. However, further studies with a larger sample size are necessary to 
strengthen the relationship of the low expression of the zinc transporters linked to the critical disease, and confirm their usefulness as a sensitive and reliable marker of zinc status.

In summary, in our study an alteration of zinc status was related with the severity-ofillness scores and inflammation in critical ill patients since admission in ICU stay. SIRS caused a general shut-down of expression of zinc transporters in whole blood. That behavior was associated with severity and inflammation of patients at ICU admission regardless zinc status. We conclude that zinc transporters in blood might be useful indicators of severity of systemic inflammation and outcome for critically ill patients.

\section{Author's Contributions}

FD, M-LJ, CH carried out the assessment, participated in the sequence alignment and drafted the manuscript. FD, EP M-LJ and IL, participated in the sequence alignment (patient's chosen, and clinical assessment conducted) and FD, M-LJ performed the statistical analysis. EP, MRE, P-CA participated in the design and conceived the study, coordinated, and helped to draft the manuscript. All authors have read and approved the final manuscript.

\section{References}

[1] C. Hogstrand, W. Maret, Genetics of Human Zinc Deficiencies, eLS. John Wiley \& Sons Ltd, Chichester, 2016.

[2] R. Robert, B. John, C. Robert, D.T. John, F. Guylaine, L. Sean, H.K. Michael, P. James, R.A. Catherine, S. Barbara, S.W. John, T.R. Judith, W.p. Keith, Z.H. Stanley, DRI - reference intake- vitamin full report, Washington, 2001.

[3] J. Abilés, A.P. de la Cruz, J. Castaño, M. Rodríguez-Elvira, E. Aguayo, R. MorenoTorres, J. Llopis, P. Aranda, S. Argüelles, A. Ayala, A.M. de la Quintana, E.M. 
Planells, Oxidative stress is increased in critically ill patients according to antioxidant vitamins intake, independent of severity: a cohort study, Crit Care 10(5) (2006) R146.

[4] B.Y. Besecker, M.C. Exline, J. Hollyfield, G. Phillips, R.A. Disilvestro, M.D. Wewers, D.L. Knoell, A comparison of zinc metabolism, inflammation, and disease severity in critically ill infected and noninfected adults early after intensive care unit admission, Am J Clin Nutr 93(6) (2011) 1356-64.

[5] A.S. Prasad, F.W. Beck, D.C. Snell, O. Kucuk, Zinc in cancer prevention, Nutr Cancer 61(6) (2009) 879-87.

[6] B. Cander, Z.D. Dundar, M. Gul, S. Girisgin, Prognostic value of serum zinc levels in critically ill patients, J Crit Care 26(1) (2011) 42-6.

[7] D.L. Knoell, M.J. Liu, Impact of zinc metabolism on innate immune function in the setting of sepsis, Int J Vitam Nutr Res 80(4-5) (2010) 271-7.

[8] M. Foster, S. Samman, Zinc and regulation of inflammatory cytokines: implications for cardiometabolic disease, Nutrients 4(7) (2012) 676-94.

[9] K. Mertens, D.A. Lowes, N.R. Webster, J. Talib, L. Hall, M.J. Davies, J.H. Beattie, H.F. Galley, Low zinc and selenium concentrations in sepsis are associated with oxidative damage and inflammation, Br J Anaesth 114(6) (2015) 990-9.

[10] J.C. King, Zinc: an essential but elusive nutrient, Am J Clin Nutr 94(2) (2011) 679S-84S.

[11] N.M. Lowe, K. Fekete, T. Decsi, Methods of assessment of zinc status in humans: a systematic review, Am J Clin Nutr 89(6) (2009) 2040S-2051S.

[12] N.M. Lowe, Assessing zinc in humans, Curr Opin Clin Nutr Metab Care (2016).

[13] L.A. Lichten, R.J. Cousins, Mammalian zinc transporters: nutritional and physiologic regulation., Annu Rev Nutr 29 (2009) 153-76.

[14] M. Foster, D. Hancock, P. Petocz, S. Samman, Zinc transporter genes are coordinately expressed in men and women independently of dietary or plasma zinc, $\mathrm{J}$ Nutr 141(6) (2011) 1195-201. 
[15] E.D. Harris, Cellular transporters for zinc., Nutr Rev 60(4) (2002) 121-4.

[16] J. Mataix Verdu, Nutricion y alimentacion Humana. vol 1 and 2, Ergon S.A. ed., Cartone, Spain, 2009.

[17] P. Singer, M.M. Berger, G. Van den Berghe, G. Biolo, P. Calder, A. Forbes, R. Griffiths, G. Kreyman, X. Leverve, C. Pichard, ESPEN, ESPEN Guidelines on Parenteral Nutrition: intensive care, Clin Nutr 28(4) (2009) 387-400.

[18] M. Gómez, I. Rodríguez, C. Cámara, M.A. Palacios, Evaluation of biological sample mineralisation methods for the determination of fluorine by graphite furnace molecular absorption spectrometry, Analyst 115(5) (1990) 553-7.

[19] J.L. English, K.M. Hambidge, Plasma and serum zinc concentrations: effect of time between collection and separation, Clin Chim Acta 175(3) (1988) 211-5.

[20] E. Fontaine, M.J. Müller, Adaptive alterations in metabolism: practical consequences on energy requirements in the severely ill patient, Curr Opin Clin Nutr Metab Care 14(2) (2011) 171-5.

[21] G.D. Phillips, V.P. Garnys, Trace element balance in adults receiving parenteral nutrition: preliminary data, J Parenter Enteral Nutr 5(1) (1981) 11-4.

[22] G.D. Phillips, V.P. Garnys, Parenteral administration of trace elements to critically ill patients, Anaesth Intensive Care 9(3) (1981) 221-5.

[23] N.Z. Cvijanovich, J.C. King, H.R. Flori, G. Gildengorin, H.R. Wong, Zinc homeostasis in pediatric critical illness, Pediatr Crit Care Med 10(1) (2009) 29-34.

[24] H.R. Wong, T.P. Shanley, B. Sakthivel, N. Cvijanovich, R. Lin, G.L. Allen, N.J. Thomas, A. Doctor, M. Kalyanaraman, N.M. Tofil, S. Penfil, M. Monaco, M.A. Tagavilla, K. Odoms, K. Dunsmore, M. Barnes, B.J. Aronow, G.o.P.S.S.S. Investigators, Genome-level expression profiles in pediatric septic shock indicate a role for altered zinc homeostasis in poor outcome, Physiol Genomics 30(2) (2007) 146-55. [25] C.J. Frederickson, W. Maret, M.P. Cuajungco, Zinc and excitotoxic brain injury: a new model, Neuroscientist 10(1) (2004) 18-25. 
[26] T. Kambe, A. Hashimoto, S. Fujimoto, Current understanding of ZIP and ZnT zinc transporters in human health and diseases, Cell Mol Life Sci 71(17) (2014) 3281-95.

[27] D. Liang, L. Xiang, M. Yang, X. Zhang, B. Guo, Y. Chen, L. Yang, J. Cao, ZnT7 can protect MC3T3-E1 cells from oxidative stress-induced apoptosis via PI3K/Akt and MAPK/ERK signaling pathways, Cell Signal 25(5) (2013) 1126-35.

[28] T. Suzuki, K. Ishihara, H. Migaki, W. Matsuura, A. Kohda, K. Okumura, M. Nagao, Y. Yamaguchi-Iwai, T. Kambe, Zinc transporters, ZnT5 and ZnT7, are required for the activation of alkaline phosphatases, zinc-requiring enzymes that are glycosylphosphatidylinositol-anchored to the cytoplasmic membrane, J Biol Chem 280(1) (2005) 637-43.

[29] E. Peters, S. Heemskerk, R. Masereeuw, P. Pickkers, Alkaline phosphatase: a possible treatment for sepsis-associated acute kidney injury in critically ill patients, Am J Kidney Dis 63(6) (2014) 1038-48.

[30] C. Lang, C. Murgia, M. Leong, L.W. Tan, G. Perozzi, D. Knight, R. Ruffin, P. Zalewski, Anti-inflammatory effects of zinc and alterations in zinc transporter mRNA in mouse models of allergic inflammation, Am J Physiol Lung Cell Mol Physiol 292(2) (2007) L577-84. 
554 Table 1. Characteristics of the study population ${ }^{1} .{ }^{1}$ Values are expressed as mean \pm 555 standard deviation. Significant differences t-test comparison: $*=p$-value $<0.05 . H S$, 556 Healthy subjects; CIP, Critical ill patients; CIPB, Critical ill patients at baseline; 557 CIPF, Critical ill patients' follow-up; APACHE II - Acute Physiology and Chronic Health Evaluation; SOFA - Sequential Organ Failure Assessment; SIRS - Systemic Inflammatory Response Syndrome.

Table 2. Biochemical values of the study population ${ }^{1} .{ }^{1}$ Values are expressed as mean \pm 562 SD. HS, Healthy subjects; CIP, Critical ill patients; CIPB, Critical ill patients at baseline; CIPF, Critical ill patients follow-up. Significant differences t-test comparison: $a=P<0.05, H S$ vs CIPB; $b=P<0.05$ CIPB vs CIPF.

Table 3. Correlation values between zinc transporters expression and severity and 567 inflammation in critically ill patients on admission and seven days in ICU stay ${ }^{1}{ }^{1}$ Values are expressed as $r$ correlation coefficient. $H S=$ Healthy subjects; $C I P$, Critical ill patients; CIPB, Critical ill patients at baseline; CIPF, Criticality ill patient's followup. Significant correlation: $a=P<0.05$. 
Fig. 1 Evaluation of mean (SD) (A) zinc supply (B) plasma zinc concentrations and (C) cellular zinc content in healthy subjects $(n=12)$ compared with critically ill patients (n $=12$ ) in intensive care unit patients at admission and seven days after ICU stay mean plasma zinc concentrations in healthy subjects $(n=12)$. Plasma and cellular zinc concentrations were determined by using ICP-MS. * Significantly differences between groups ( $\mathrm{P}<0.05$, $\mathrm{t}$-student test comparison).

Fig. 2 (A) Heat map showing an overview of the Ct quality categories that have been assigned to the different zinc transporters. The categories correspond to "High expression level", "Middle expression level" and "Undetermined" with the colour codes "Red", "Green-Blue" and "Black" respectively, in healthy subjects (HS) (n=12) and critically ill patients $(\mathrm{n}=12)$ at admission $(\mathrm{CIPB})$ and seven days of ICU stay (CIPF). (B) RNA expression critical ill patients at baseline in intensive care unit vs healthy subjects. (C) RNA expression critical ill patients at baseline in intensive care unit vs critical ill patients at baseline in intensive care unit. $\Delta \mathrm{Ct}=\mathrm{Ct}$ (cycle threshold) target Ct reference. Statistically significant differences: $*=\mathrm{P}<0.05 ; * *=\mathrm{P}<0.01$.

Fig. 3 (A) Evaluation of mean (SD) of albumin, (B) plasma zinc:albumin ratio and (C) cellular zinc:albumin ratio in healthy subjects $(\mathrm{HS})(\mathrm{n}=12)$ compared with critically ill patients $(\mathrm{n}=12)$ in intensive care unit at admission (CIPB) and seven days (CIPF). Box-and-whisker plots show median, interquartile and full range. * Significantly differences between groups ( $\mathrm{P}<0.05$, $\mathrm{t}$-student test comparison).

Fig. 4 Comparative analysis of zinc status and zinc transporters by severity and inflammation in critically illness. Severity scores including APACHE and SOFA, and inflamation parameters like CRP were dicotomized by the median value. (A) Plasma zinc concentrations according to SOFA score and CRP levels in critically ill patients 
during the follow-up study $(\mathrm{n}=12)$. (B) RNA expression of ZIP3 by low APACHE vs 600 high APACHE and low CRP vs high CRP in critically ill patients on ICU admission. 601 (C) RNA expression of ZnT6 and ZnT by low APACHE vs high APACHE in critically 602 ill patients on admission in ICU stay. (D) RNA expression of ZIP4 and ZIP8 by low 603 CRP vs high CRP in critically ill patients on admission and within the follow-up study. 604 Box-and-whisker plots show median, interquartile and full range. Box-and-whisker 605 plots show median, interquartile and full range. $\Delta \mathrm{Ct}=\mathrm{Ct}$ (cycle threshold) target $-\mathrm{Ct}$ 606 reference. * Significantly differences between groups $(\mathrm{P}<0.05$, t-student test 607 comparison).

608

609 Fig. 5 RNA expression of ZIP8 in healthy controls (HS) and critically ill patients on 610 admission (CIPB) and within the follow-up study (CIPF) $(n=12)$. Box-and-whisker plots 611 show median, interquartile and full range. $\Delta \mathrm{Ct}=\mathrm{Ct}$ (cycle threshold) target $-\mathrm{Ct}$ 612 reference. * Significantly differences between groups $(\mathrm{P}<0.05$, t-student test 613 comparison). 


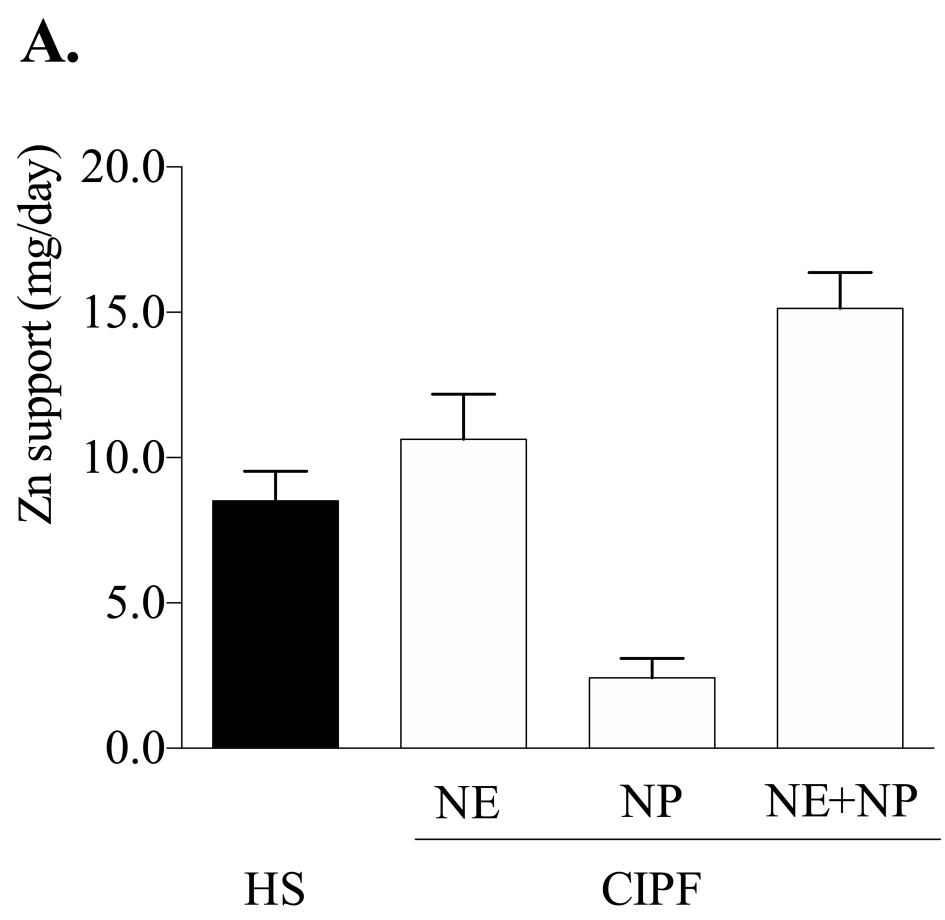

B.

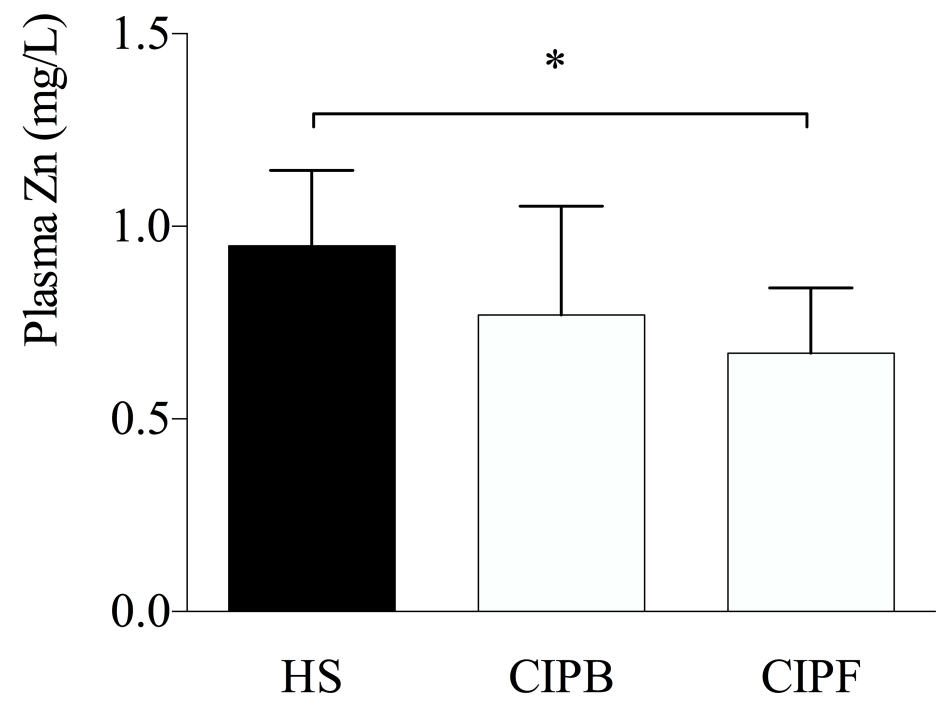

C.

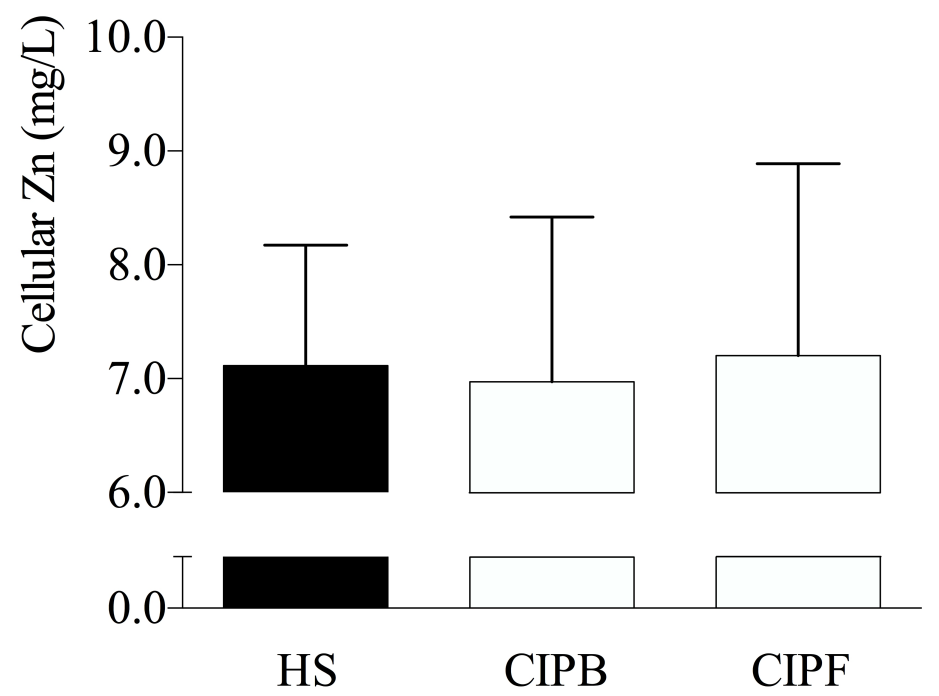




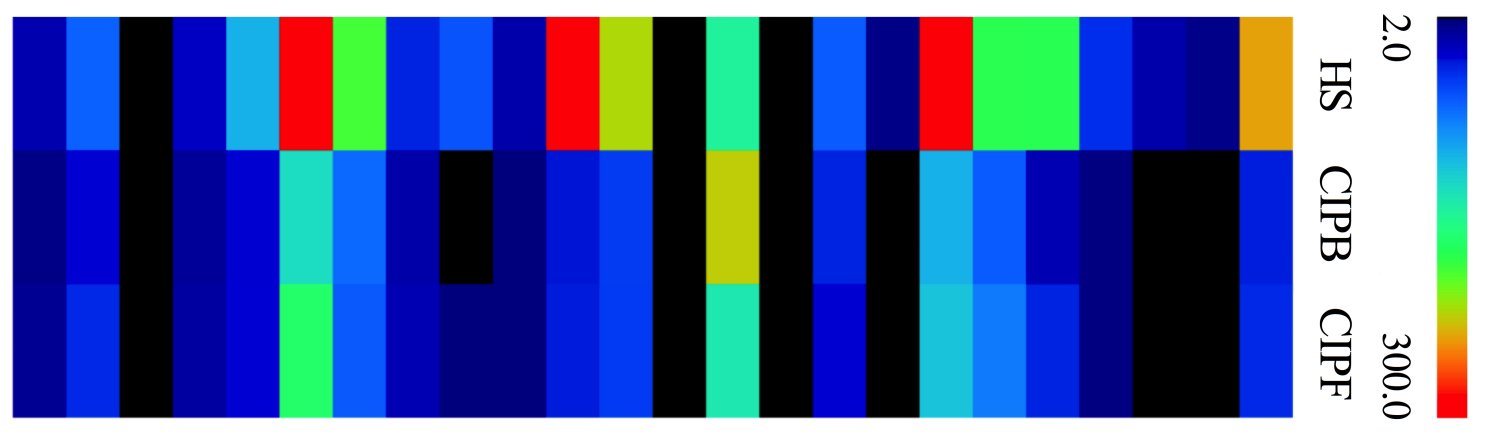

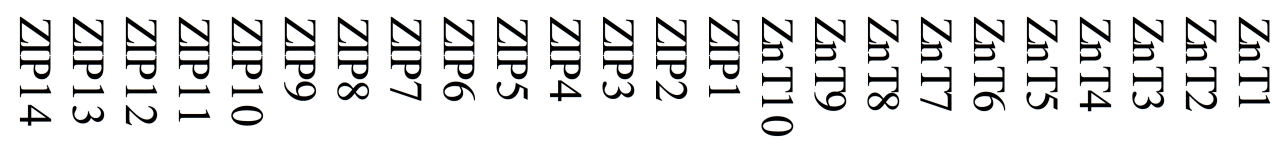

?

to

$\log 2 \mathrm{FD}$
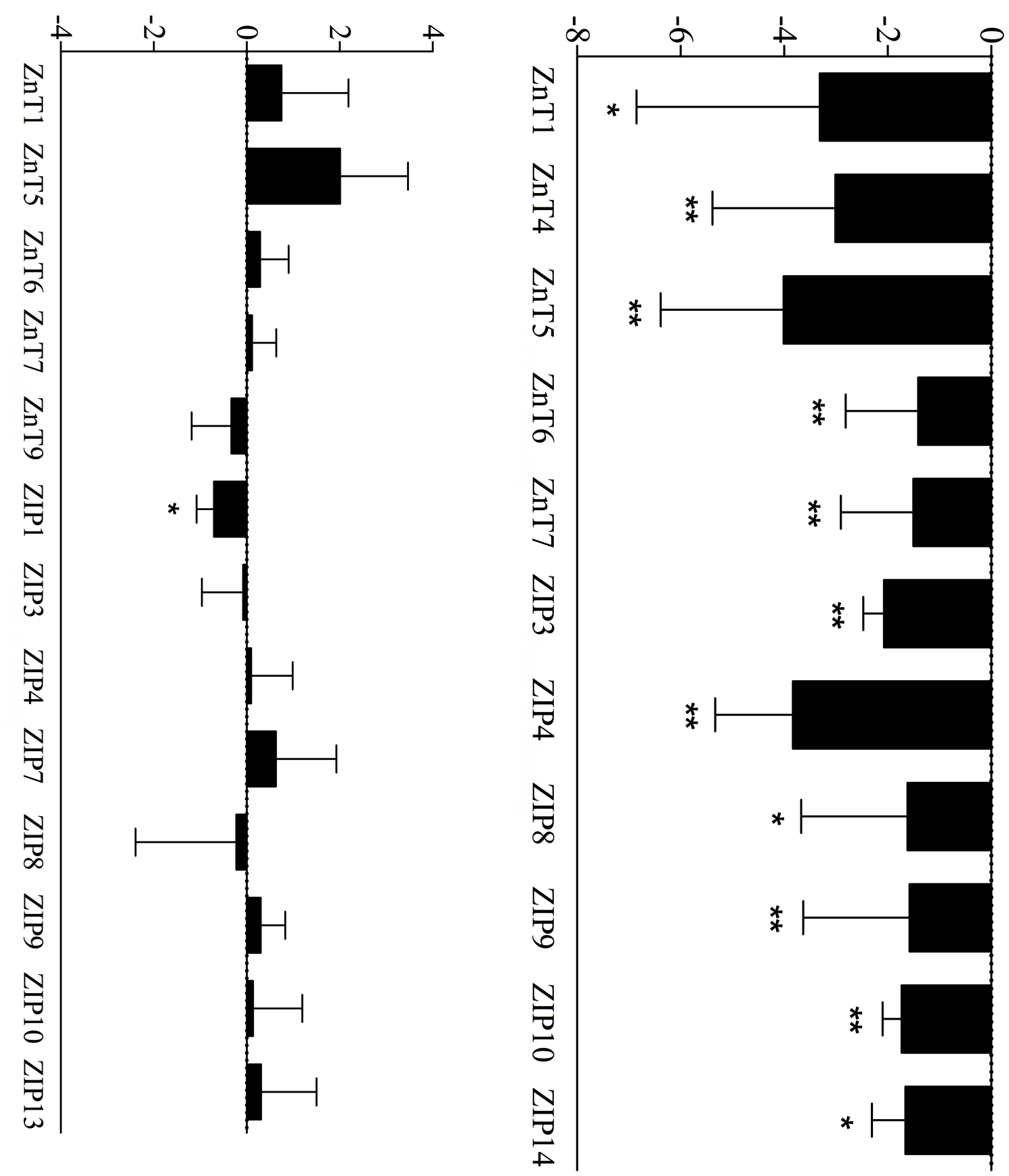


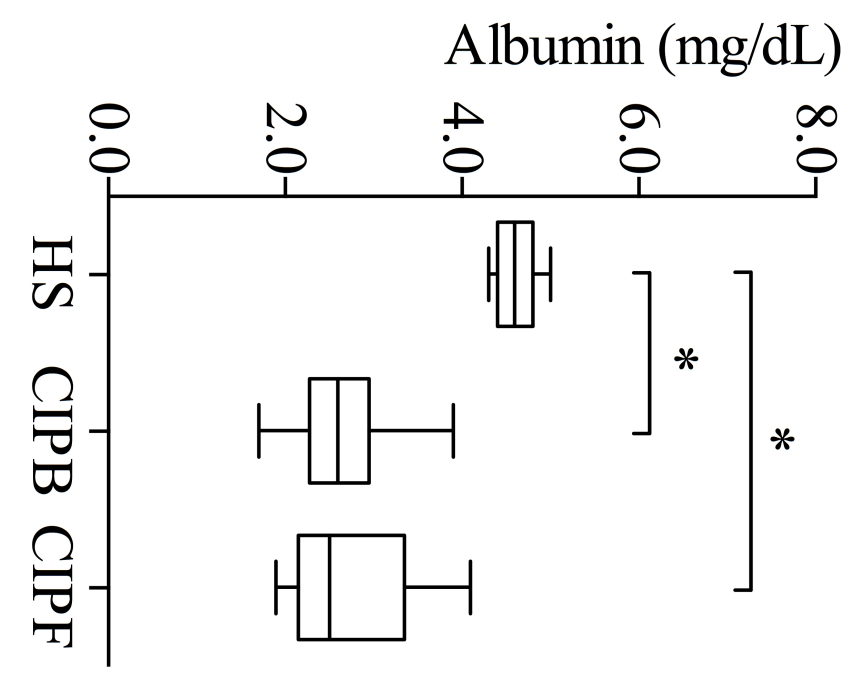

$\varpi$

Plasma Zn / Albumin ratio

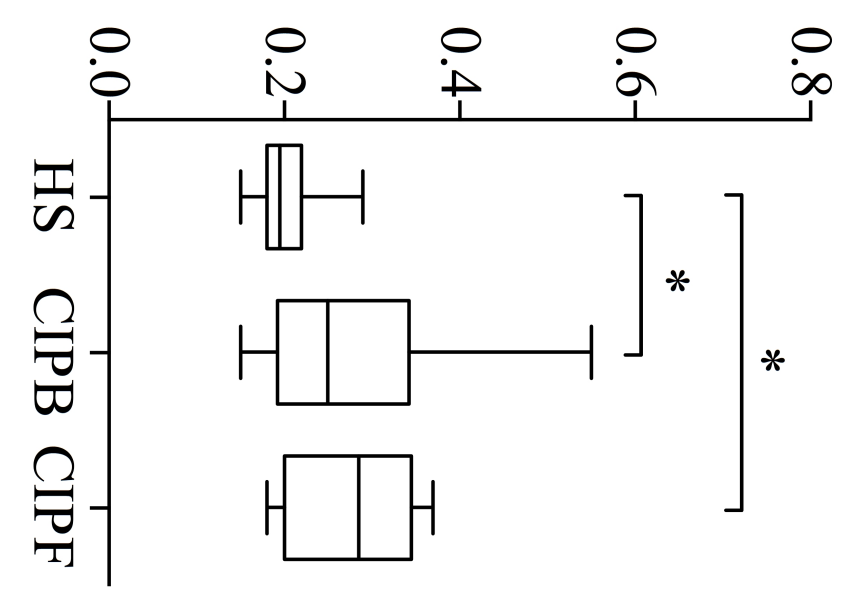

$\Omega$

Cellular Zn / Albumin ratio

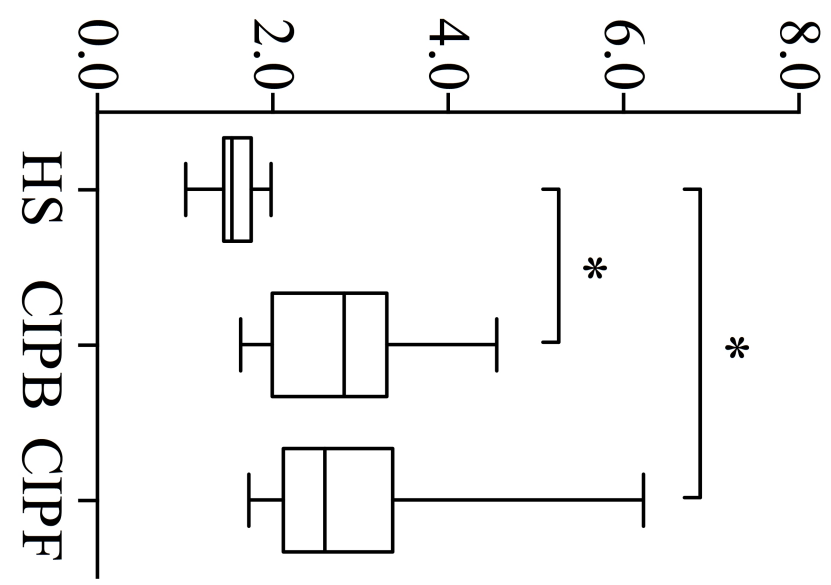



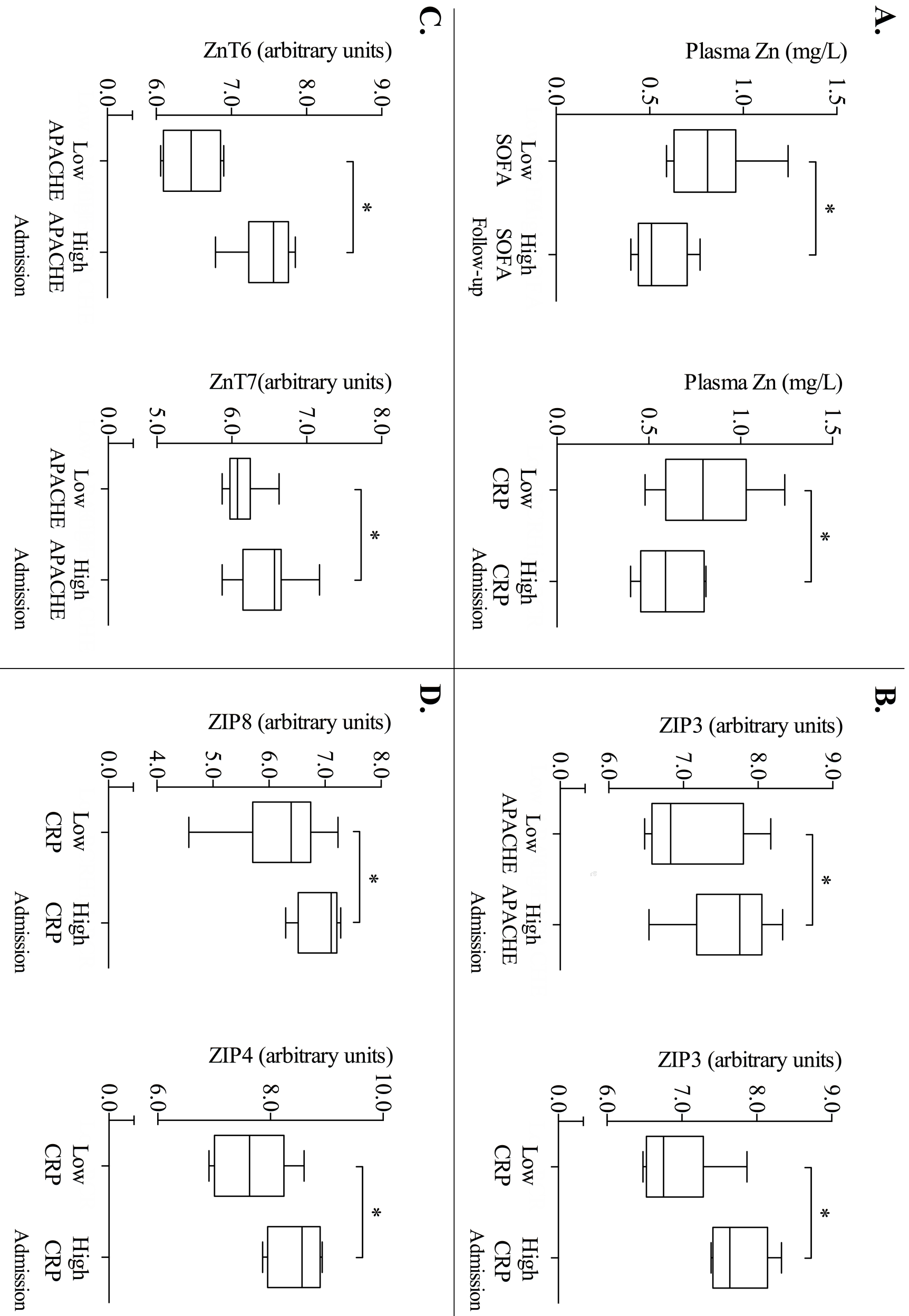


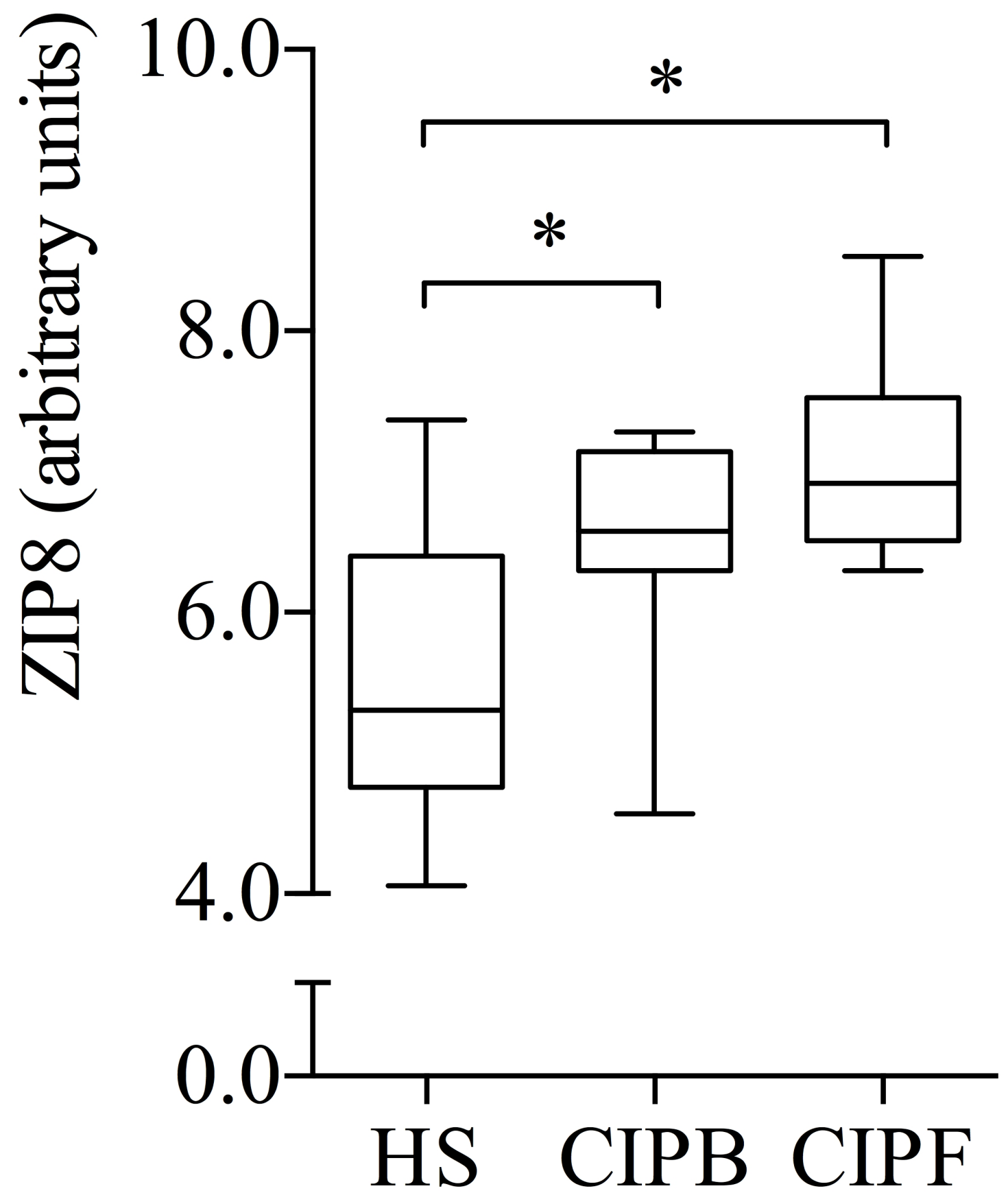


Table 1. Characteristics of the study population ${ }^{1}$.

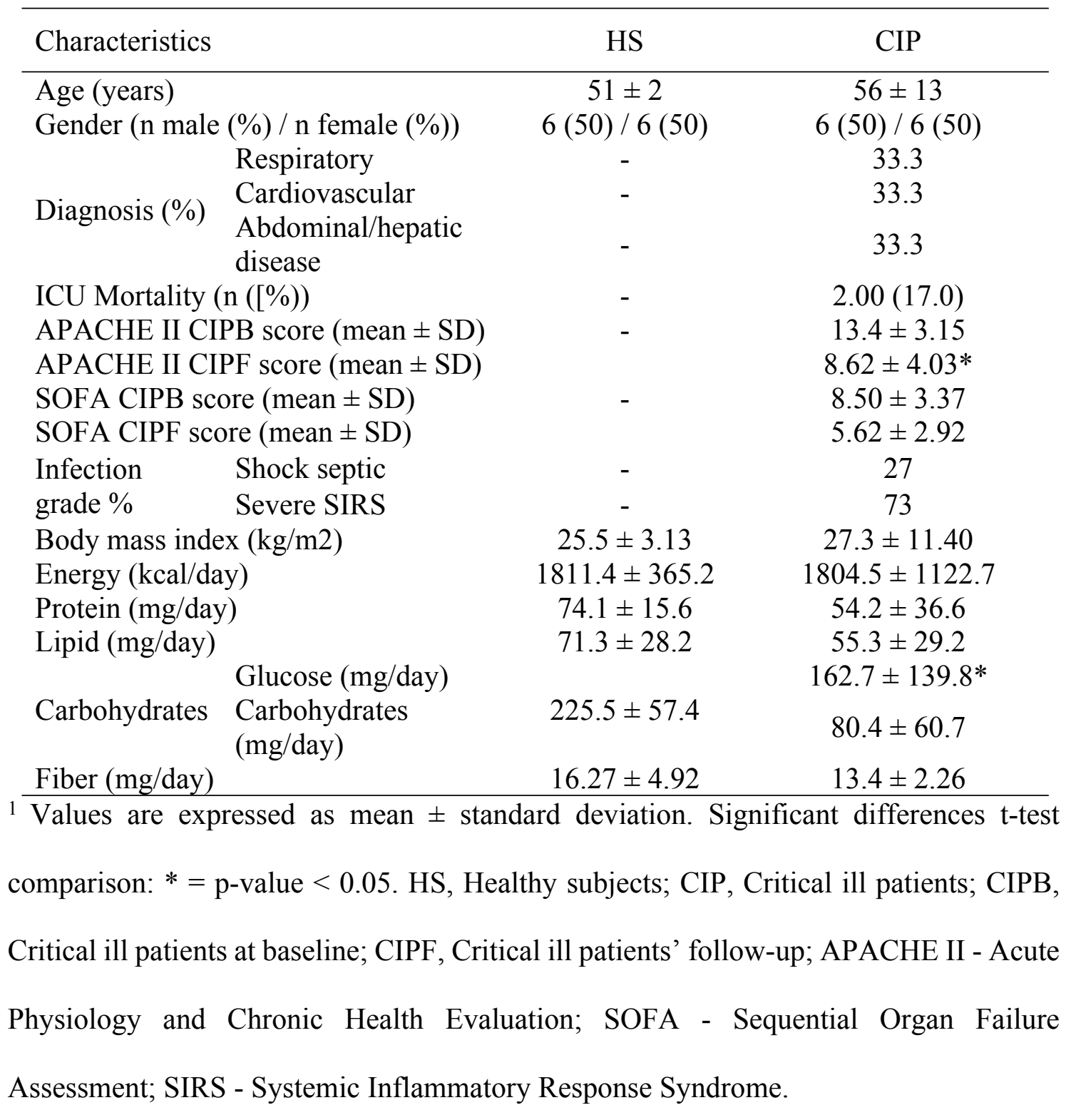


Table 2. Biochemical values of the study population ${ }^{1}$.

\begin{tabular}{|c|c|c|c|}
\hline Characteristics & HS & CIPB & CIPF \\
\hline Glucose (mg/dL) & $93.5 \pm 25.2$ & $171.5 \pm 118.5^{\mathrm{a}}$ & $167.5 \pm 37.8$ \\
\hline Albumin $(g / d L)$ & $4.59 \pm 0.21$ & $2.6 \pm 0.60^{\mathrm{a}}$ & $2.7 \pm 0.71$ \\
\hline Pre-albumin (mg/dL) & $25.3 \pm 9.78$ & $11.2 \pm 4.85^{\mathrm{a}}$ & $19.4 \pm 11.5$ \\
\hline Urea $(\mathrm{mg} / \mathrm{dl})$ & $33.5 \pm 9.07$ & $68.5 \pm 42.2^{\mathrm{a}}$ & $59.1 \pm 31.5$ \\
\hline Uric acid $(\mathrm{mg} / \mathrm{dl})$ & $4.47 \pm 1.11$ & $4.44 \pm 3.44$ & $2.06 \pm 0.77^{\mathrm{b}}$ \\
\hline Alkaline phosphatase (U/L) & $69.6 \pm 19.9$ & $84.7 \pm 66.1$ & $127.3 \pm 105.2$ \\
\hline Creatinine $(\mathrm{mg} / \mathrm{dl})$ & $0.77 \pm 0.17$ & $1.39 \pm 1.32$ & $0.77 \pm 0.50^{\mathrm{b}}$ \\
\hline $\mathrm{CPK}(\mathrm{U} / \mathrm{L})$ & $84.5 \pm 32.8$ & $978.1 \pm 1271.1^{\mathrm{a}}$ & $368.1 \pm 514.4$ \\
\hline C Reactive Protein (mg/dl) & $0.21 \pm 0.11$ & $15.1 \pm 7.65^{\mathrm{a}}$ & $8.33 \pm 5.38^{b}$ \\
\hline Rheumatoid factor (UI/ml) & $6.66 \pm 2.46$ & $10.9 \pm 4.48^{\mathrm{a}}$ & $11.4 \pm 7.90$ \\
\hline Total protein $(\mathrm{g} / \mathrm{dl})$ & $7.16 \pm 0.47$ & $4.86 \pm 0.70$ & $5.28 \pm 0.66$ \\
\hline Transferrin $(\mathrm{mg} / \mathrm{dl})$ & $267.7 \pm 78.7$ & $136.5 \pm 93.1^{\mathrm{a}}$ & $133.5 \pm 58.2$ \\
\hline Leucocyte $\left(10^{\wedge} 3 \mathrm{microl}\right)$ & $6.20 \pm 1.39$ & $14.6 \pm 4.65^{\mathrm{a}}$ & $11.7 \pm 4.51$ \\
\hline Copper $(\mathrm{Cu})(\mu \mathrm{g} / \mathrm{mL})$ & $83.1 \pm 12.7$ & $82.2 \pm 29.4$ & $80.6 \pm 23.1$ \\
\hline Iron $(\mathrm{Fe})(\mu \mathrm{g} / \mathrm{mL})$ & $128.8 \pm 44.2$ & $31.7 \pm 21.9^{a}$ & $40.4 \pm 32.6$ \\
\hline Homocysteine $(\mu \mathrm{mol} / \mathrm{L})$ & $20.8 \pm 16.2$ & $11.2 \pm 6.4$ & $9.57 \pm 2.82^{\mathrm{a}}$ \\
\hline
\end{tabular}




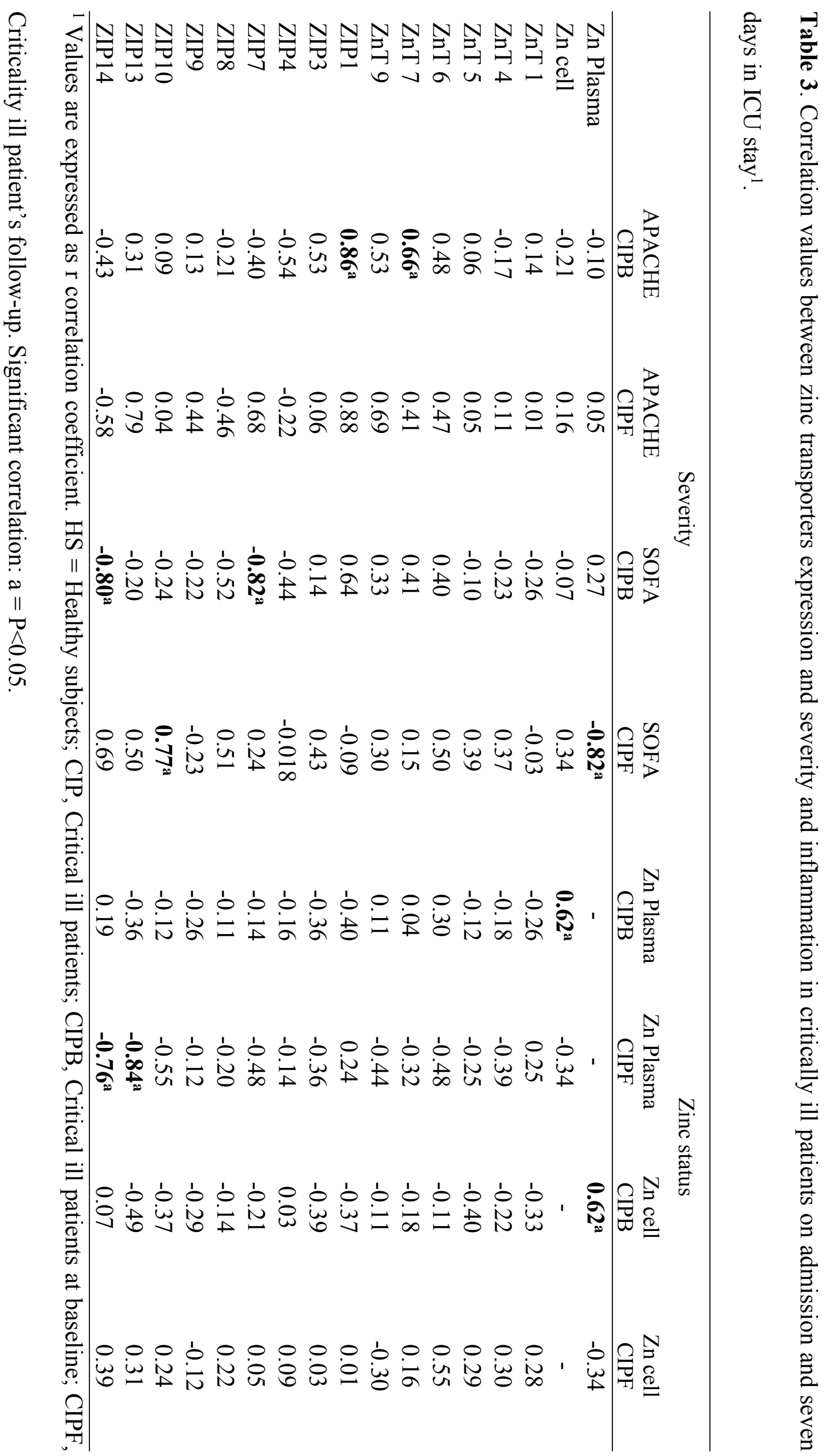




\section{Conflicts of Interest}

The authors declare no conflict of interest. 